\title{
Auto-tuning Fractional Order Control of a Laboratory Scale Equipment
}

\author{
Rusu-Both Roxana* and Dulf Eva-Henrietta \\ Automation Department, Technical University of Cluj-Napoca, Memorandumului Street, No.28 Cluj-Napoca, Romania \\ ${ }^{*}$ Corresponding author
}

\begin{abstract}
Distributed control systems with high nonlinearity and with uncertain characteristics, make the control development a very complex task. In the last period several control methods were developed, presenting different performances but there is still room for improvement. In order to overthrow the complexity of the process a possible approach is to use robust control methods. To this end, the research paper proposes fractional order parameter tuning adaptive algorithm to maintain the output value and to fulfill the imposed requirements. The performances of the proposed control system are verified through digital simulations using Matlab/SIMULINK environment and were validated in real time applications using a laboratory experimental setup.
\end{abstract}

Keywords-anti-lock braking system; fractional order control; adaptive control; nonlinear model; wheel slip

\section{INTRODUCTION}

It is a well-known fact that in industrial application the classical PID controller is used in $99 \%$ of the cases even if several, more advanced, control techniques are available and could give better performance results. This happens due to the simplicity and the effectiveness of the PID controller which makes it easier to understand and tune by the operators. In 1999 Podlubny [7] presented the fractional order controller as a generalization of the classical PID controller by using any real order instead of only integer order for the integration and differentiation introducing in this manner 2 extra degrees of freedom making possible to introduce additional specification in the design. The number of applications of fractional calculus increased considerably in the last decade due to the development of more advanced mathematical computational tools. The advantages of fractional order controllers with respect to classical PID controller were clearly demonstrated in different applications like DC motor control [1], service robot control [11], electro-hydraulic system [13], chemical processes $[2,3]$. The key problem is that in order to be able to tune the fractional order control parameters a basic knowledge of fractional calculus is needed $[9,12]$ being the reason why most engineers are reluctant in using them.

The increased complexity of controller parameter tuning process lead to an increased interest in auto-tuning control methods which are able to handle even process variations, in case of infrastructure problems of the control system, instrumentation problems or even non-critical faults. One of the widely used methods in industrial applications is the relay autotuning method. This method can be extended even in the case of fractional-order controllers [8]. Unfortunately most existing fractional order design methods are complex which represents an important issue in any auto-tuning method.

The present work proposes a novel, simplified algorithm for fractional order controller parameter tuning included in an auto-tuning control scheme. The antilock brake system was chosen as case study to validate the proposed control system representing a great challenge from the control point of view due to the complex and nonlinear braking dynamics.

The paper is structured in four parts. After the introductory part, the proposed fractional order controller design method is presented in the second section together with the auto-tuning control scheme while the third section details the proposed case study, with the experimental results and validation. The work ends with concluding remarks.

\section{Auto-tuning Fractional ORder CONTROL SyStem}

As presented in the introductory part the fractional order controller can be considered as a generalization of the classical PID controller having the following form $[8,10]$ :

$$
H_{F O-P I D}(s)=K_{P}\left(1+\frac{K_{i}}{s^{\lambda}}+K_{d} s^{\mu}\right), \lambda, \mu \in \mathfrak{R},
$$

where $\mathrm{KP}, \mathrm{Ki}, \mathrm{Kd}$ are the classical tuning parameters of a PID controller and $\lambda$ and $\mu$ are the integration and differentiation orders.

The tuning process of the fractional order PID controllers (FOPID) is in fact reduced to determining the five tuning parameters to ensure the imposed performance criteria. The explicit form of the most used performance criteria in frequency domain are [8]:

- The phase margin $\varphi_{\mathrm{m}}$ used to impose a certain close loop overshoot

$$
\left|H_{R}\left(j \omega_{g c}\right) \cdot H_{P}\left(j \omega_{g c}\right)\right|=0 d B
$$

- The gain crossover frequency, $\omega$ gc,used to ensure a certain closed loop settling time.

$$
\arg \left(H_{R}\left(j \omega_{g c}\right) \cdot H_{P}\left(j \omega_{g c}\right)\right)=-\pi+\varphi_{m}
$$


- The iso-damping property, used to ensure a constant overshoot of the closed loop system, even in the presence of open loop gain variations.

$$
\left.\frac{d \arg \left(H_{R}(j \omega) \cdot H_{P}(j \omega)\right)}{d \omega}\right|_{\omega=\omega_{c g}}=0
$$

where HR(s) represents the controller transfer function and $\mathrm{HP}(\mathrm{s})$ represents the process transfer function. Other performance criteria can be considered [8] like high frequency noise rejection condition or good output disturbance rejection condition depending of the application in question. It must be mentioned that determining the controller tuning parameters is not an easy task being needed to solve the system of nonlinear equations resulted from the imposed performance criteria.

For simplicity reasons, the fractional order PI controller design method is firstly presented having the following transfer function:

$$
H_{F O-P I}(s)=K_{P}\left(1+\frac{K_{i}}{s^{\lambda}}\right), \lambda, \mu \in \mathfrak{R}
$$

Based on the previously presented performance criteria and the explicit form from eq.(2-4), an attempt to analytically determine the controller tuning parameters was made. An iterative algorithm was proven to be the simplest solution as follows:

Step $0 \quad \lambda=1$;

Step 1 determine Ki using eq. (6-11)

Step 2 determine Kp using eq. (12)

Step 3 check if the imposed performance are met

if yes then the design is finished

else $\lambda=\lambda-\Delta \lambda$ and RETURN TO STEP 1

$$
\begin{gathered}
a=\sin \frac{\lambda \pi}{2} ; b=\cos \frac{\lambda \pi}{2} ; \quad\left|H_{p}(j \omega)\right|=\frac{1}{\sqrt{K^{2}+L^{2}}} \\
x 1=2 \cdot L \cdot P \cdot \omega_{g c}^{\lambda+1} ; x 2=2 \cdot K \cdot Q \cdot \omega_{g c}^{\lambda+1} ; \\
x 3=K^{2} \cdot \lambda \cdot \omega_{g c}^{\lambda} ; x 4=L^{2} \cdot \lambda \cdot \omega_{g c}^{\lambda} \\
x 5=-4\left(L P \omega_{g c}-K Q \omega_{g c}\right)\left(L P \omega_{g c}^{2 \lambda+1}-K Q \omega_{g c}^{2 \lambda+1}\right) \\
x 6=x 1 \cdot b+x 2 \cdot b-x 3 \cdot a-x 4 \cdot a \\
K_{I}=\frac{x 6 \mp \sqrt{x 5+x 6^{2}}}{2\left(L P \cdot \omega_{g c}-K Q \cdot \omega_{g c}\right)} \\
K_{P}=\mp \frac{\omega_{g c}^{\lambda} \sqrt{K^{2}+L^{2}}}{\sqrt{K_{I}^{2}+\omega_{g c}^{2 \lambda}+2 \cdot K_{I} \cdot \omega_{g c}^{\lambda} \cdot b}}
\end{gathered}
$$

The designed fractional order controller can be included in an auto-tuning control structure [6] as the one presented in Figure I:

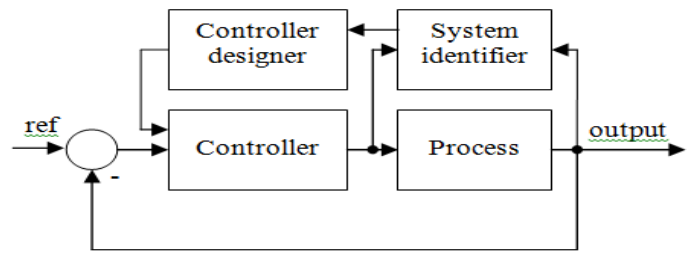

\section{FIGURE I. AUTO-TUNING CONTROL STRUCTURE}

The idea behind this control is to create a closed loop controller with parameters that can be updated as the process parameters vary. As it can be observed from the control structure the process is identified each time step by the "System identifier" block which uses a recursive simple least-squares (RLS) algorithm to estimate the system parameters [6]. The "Controller designer" block implements the previously presented algorithm and the fractional order controller parameters are tuned based on the new parameter values of the process. In the case of fractional order controllers the operators are defined by irrational continuous transfer functions, in the Laplace domain which often causes evaluation problems in the simulations. Therefore, continuous or discrete integer-order approximations of the fractional order operators need to be adopted when analyzing fractional order systems. The recursive equation corresponding to the designed fractional order controller is implemented in the "Controller" block using the Oustaloup-Recursive-Approximation [5].

\section{Auto-TUNing Fractional ORDER SLip CONTROL OF ABS SYSTEM}

The proposed auto-tuning fractional order control structure is used for slip control for a laboratory anti-lock brake system. The experimental unit is anti-lock brake system designed by Inteco [7], Figure II, in order to optimize braking effectiveness while maintain the car controllability. This laboratory set-up is able to test the performance of the ABS system for various road conditions for example wet asphalt.

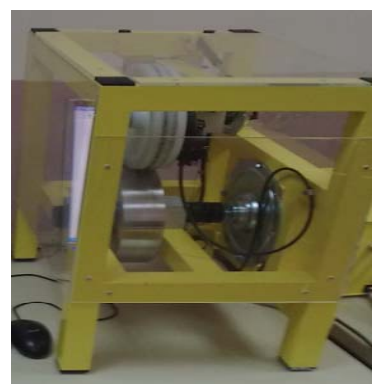

FIGURE II. THE ANTI-LOCK BRAKE SYSTEM BY INTECO

The main components [7] of the experimental unit are: two rolling wheels, a flat $\mathrm{DC}$ motor used to drive the ABS, two identical encoders used to measure the rotational angles of the wheels with a high resolution 4096 pulses per revolution. The lower wheel simulates the relative road motion while the upper wheel mounted on a lever simulates the car wheel. The PC is 
equipped with a RT-DAC/PCI-D multipurpose digital I/O board which communicates with the power interface. The power interface amplifies the control signals received from the PC and transmits them to the DC motor. The encoder pulse signals are converted to digital 16-bit form and transmitted to the PC using the same power interface. All the functions of the board can be accessed using the ABS Toolbox directly from Matlab/SIMULINK environment. The Simulink implementation of the proposed control scheme is presented in Figure III. and all the block elements from Figure II can be identified.

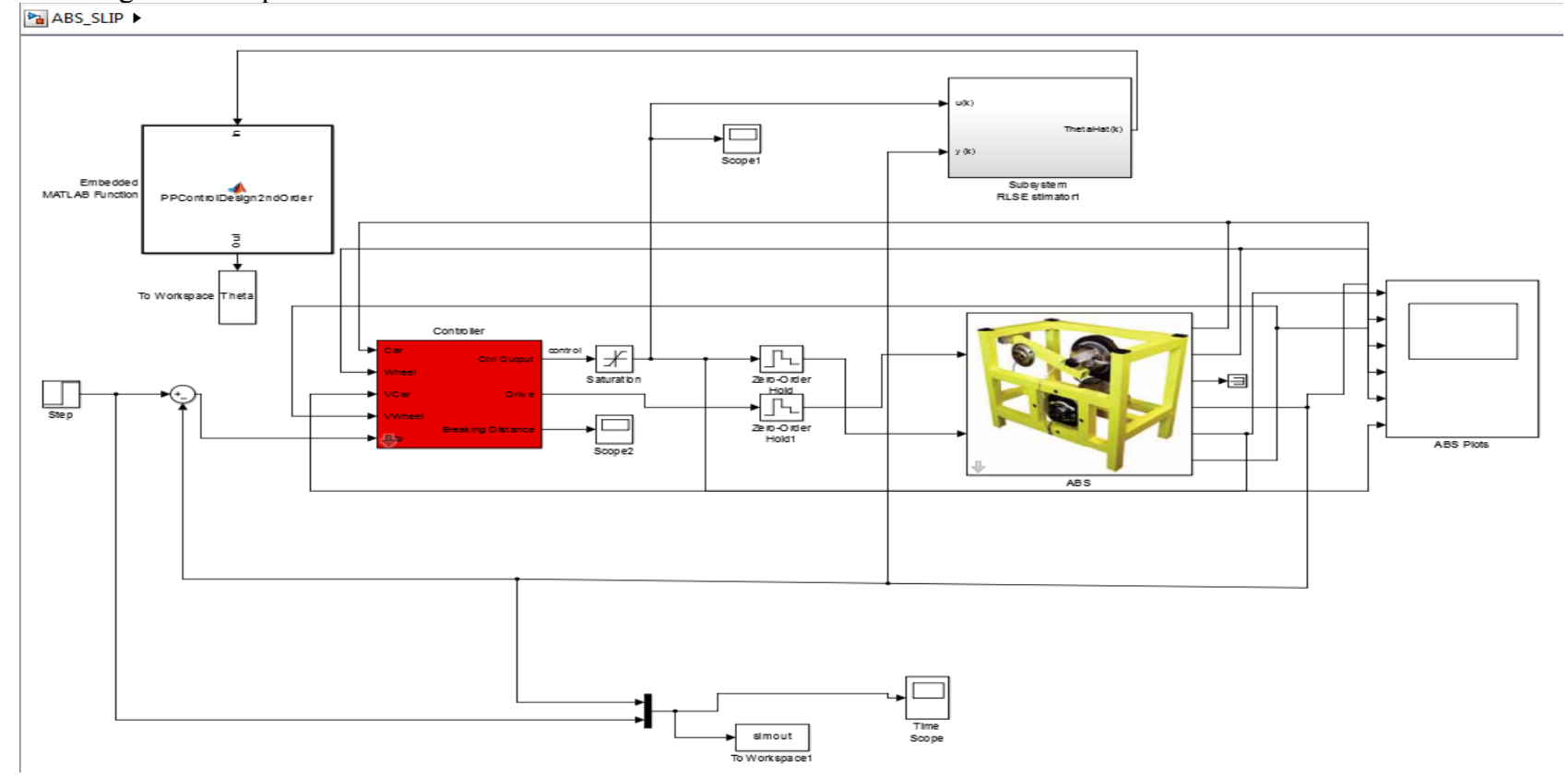

FIGURE III. THE SIMULINK IMPLEMENTATION OF THE AUTO-TUNING FRACTIONAL ORDER CLIP CONTROL STRUCTURE FOR THE ANTI-LOCK BRAKE SYSTEM

The main objective of the ABS control system is to prevent wheel blocking during the braking process otherwise known as wheel-lock. This phenomenon can be observed by monitoring the wheel slip, in this case considered as the relative difference of the wheels velocities. The wheel slip needs to be controlled so that the friction coefficient between the car and the road is maximized. The control structure is implemented in real time. The process is identified each time step as a second order system and the fractional order controller is approximated with Ouastaloup recursive method [5] as a second order transfer function. If the approximation order is increased the accuracy will also increase. However, in an auto-tuning control scheme the computations of higher complexity are to be avoided if possible.

The experimental procedure consists in accelerating the wheel that animates the car-road motion to an imposed threshold velocity using the DC motor. The next phase of the experimental procedure is the braking phase, when the DC motor is switched off to enable the free motion of both wheels. In this phase the brake is activated until the car velocity achieves the zero value while keeping the wheel from slipping.

This experimental procedure using the proposed control structure was tested in different road conditions. For the first test a perfectly dry asphalt road was considered, then for the second test a bumpy dry road and lastly the worst case scenario was considered: wet asphalt.
The experimental results for the first test are presented in Figure IV.A) The car position, wheel position, slip, car velocity, wheel velocity and brake evolutions can be observed in this order from top to bottom of the figure. The considered value for the imposed threshold velocity for the first phase of the experimental procedure is $2000 \mathrm{rpm}$ and will be kept for all the other experimental tests. It can be observed that the wheel and the car reach the imposed velocity after around 8 seconds when the braking phase will start.

By analyzing the evolution of the wheel and car velocity we can determine that the car stops after approximately 4.4 seconds. Another important parameter evolution is the evolution of the slip which in this case doesn't increase above a 0.05 value.

In the second experimental test a bumpy road was simulated. The experimental results can be observed in Figure IV.B) In this case the car and wheel reach the imposed threshold velocity at around the same time as in the previous case, the slip value increased to 0.06 only for a short instant and the car reaches to stop at 12.7 seconds. The car and the wheel positions are relatively the same as in the previous test so it can be concluded that the proposed control structure performances for rough road conditions are maintained.

The last test experiment was designed to determine the performance of the designed control structure in worst case conditions meaning wet asphalt. 

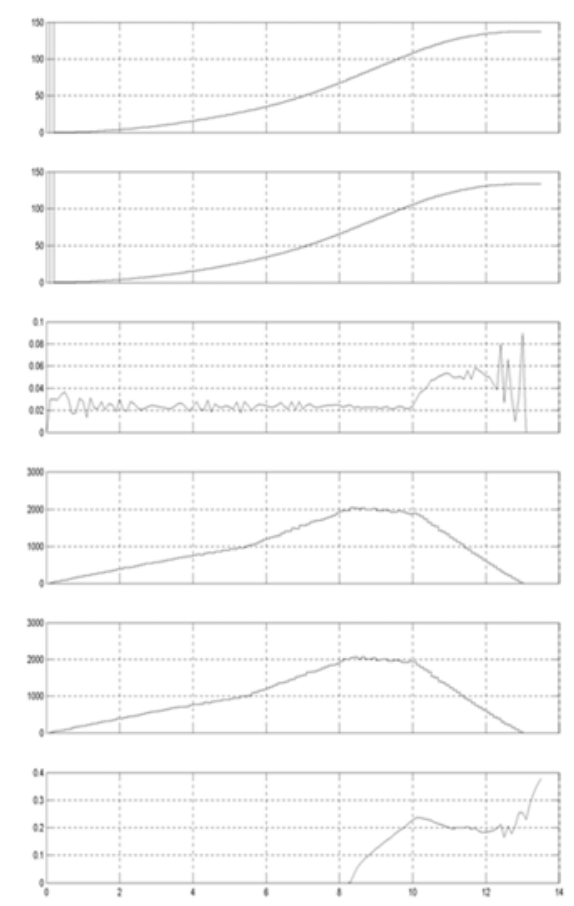

tremente

A) DRY ASPHALT
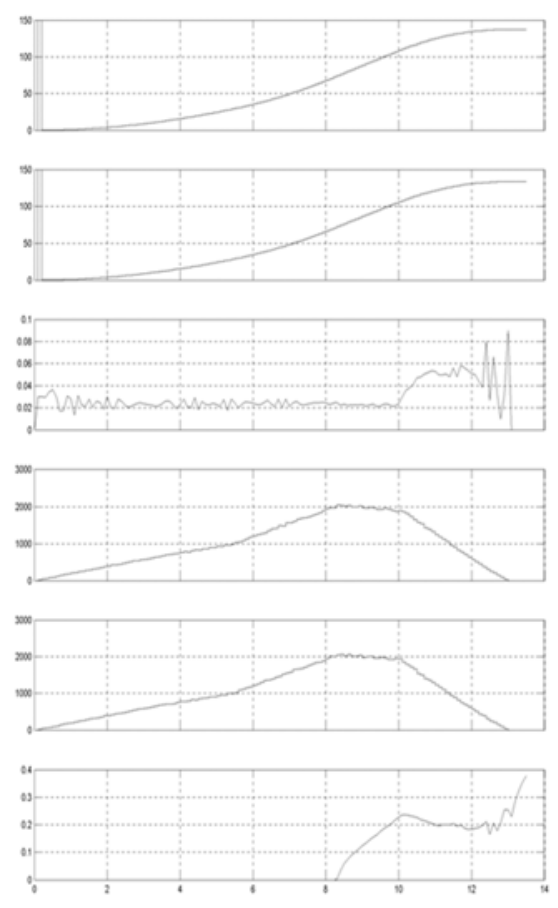

B) BUMPY ROAND
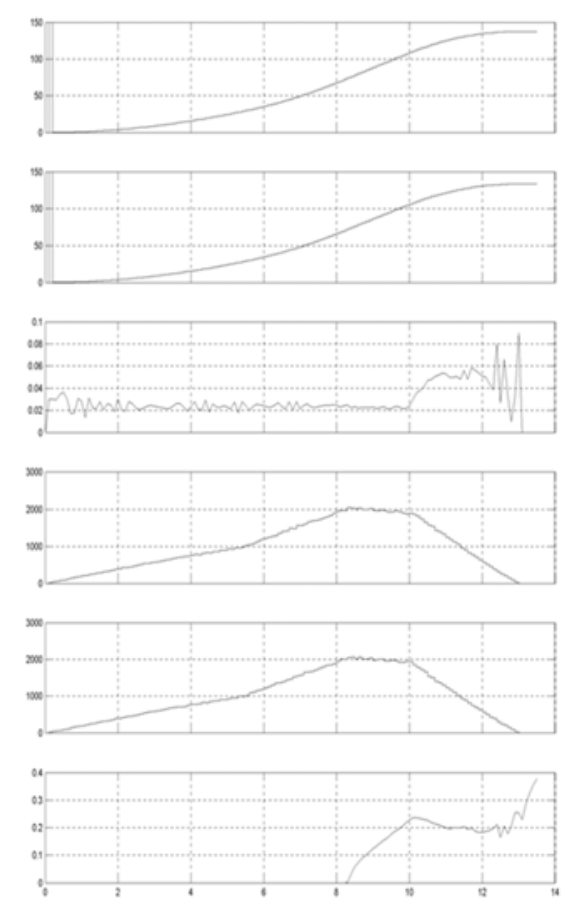

tenome

C) WET ASPHALT

FIGURE IV. EXPERIMENTAL RESULTS OF THE AUTO-TUNING FRACTIONAL ORDER SLIP CONTROL STRUCTURE OF THE ABS SYSTEM

The experimental results are presented in Figure IV.C) In this case the evolutions of the brake and of the slip are quite different in comparison to the other experimental tests. The slip reaches value around 0.08 and the control signal, the brake is much more active. The braking time increases from 4.4 seconds (dry asphalt) to 4.8 seconds.

This increase was expected but considering that the car and wheel positions are relatively the same it can be concluded that the proposed control solution performs in a satisfactory manner even for worst case road conditions.

\section{CONCLUSIONS}

An auto-tuning structure of a fractional order controller has been proposed. The idea was to get the benefit from the high performance quality of fractional order systems confirmed in many precedent research works. The control structure is exemplified on the slip control of a laboratory anti-lock braking system which represents a great challenge from the control point of view due to the complex and nonlinear braking dynamics. The experimental test results show that the proposed tuning technique is accurate and produces robust closed loop results. They also revealed that this combined method main benefit consists in the evolution of the control signal. 


\section{ACKNOWLEDGMENT}

This work was supported by a grant of the Romanian

National Authority for Scientific Research and Innovation, CNCS- UEFISCDI, project number 155/2012 PN-II-PT-PCCA.

\section{REFERENCES}

[1] B.B. Alagoz, Ates A,Yeroglu C. "Auto-tuning of PID controller according to fractional-order reference model approximation for DC rotor control", Mechatronics,vol.23 (issue 7), pp.789-797, 2013

[2] R Both, Cormos A, Agachi S, Festila C, Dynamic modeling and validation of 2-ethyl-hexenal hydrogenation process, Computers\&Chemical Engineering, 52, 100-111, 2013

[3] R Both, Dulf E, Festila C, Dynamic modeling and validation of 2-ethylhexenal hydrogenation process, Chemical Eng. Sci., 74, 300-309, 2012

[4] R Both et. all, Microcontroller implementation of Digital Fractional Order Controllers for Time Delay Processes, 21st Mediterranean Conference on Control and Automation (MED), 1445-1450, 2013

[5] Y.Q. Chen,'Oustaloup-Recursive-Approximation for Fractional Order Differentiators", $\quad$ Updated $\quad 16 \quad$ Feb 2014 http://www.mathworks.com/matlabcentral/fileexchange/authors/9097

[6] S.Haykins.”Adaptive Filter Theory", Prentice Hall, 2002

[7] Inteco, “Anti-Lock Brake System -User's Manual”, available at www.inteco.com.pl, accessed 2016

[8] C.A.Monje, B.Vinagre, Feliu M.F., Chen.Y.Q "Tuning and autotuning of fractional order controllers for industry applications",Control Engineering Practice 16, pp. 798-812, 2008

[9] F. Padula, A.Visioli, "Advances in robust fractional control", Springer, ISBN 978-3-319-10929-9, 2014

[10] I. Podlubny,"Fractional-ordersystems and PI $\lambda \mathrm{D} \mu$-controllers", IEEE Transactions on Automatic Control 44, 208-214, 1999

[11] T.Qingshun, Chunfu W, Yuanhui Y, Guodong L, Fengyu Z.,’Design and Implementation of Fractional Order Controller for Service Robots", International Journal of Control and Automation, Vol.8, No.5, 2015

[12] D Xue et. all, "Linear Feedback Control Analysis and Design with MATLAB", Advances in Design and Control, Siam, 2007

[13] J.Zhao, Wang J., Wang S., "Fractional order control to the electrohydraulic system in insulator fatigue test device", Mechatronics, 2013 